\title{
Neural Backpropagation System for the Study of Obesity in Childhood
}

\author{
A. Medina-Santiago \\ Dept. Center of Investigation, Development and Innovation \\ Technology (Cidit) \\ University of Science and Technology Descartes \\ Tuxtla Gutierrez, Chiapas, Mexico
}

\author{
E. M. Melgar-Paniagua \\ School Science Biology \\ Institute Polytechnic National \\ Mexico City, Mexico
}

\author{
L. C. Campos-Reyes \\ Nutrition Faculty \\ University of Veracruz \\ Veracruz, Mexico
}

\author{
A. Cisneros-Gómez, N. R. García-Chong \\ Dept. Center of Investigation, Development and Innovation \\ Technology (Cidit), University Autonomy of Chiapas, \\ Faculty Medicine \\ University of Science and Technology Descartes \\ Tuxtla Gutierrez, Chiapas, Mexico
}

\begin{abstract}
This paper presents the development of a nutritional system using Backpropagation neural network, that is able to provide a clear and simple prediction problems of obesity in children up to twelve years, based on your eating habits during the day. For the development of this project has taken into account various factors, which are vital for the proper development of infants. A prediction system can offer a solution to several factors, which are not easily determined by convectional means.
\end{abstract}

Keywords-prediction system; Nutrition; Backpropagation Neural Network; Obesity

\section{INTRODUCTION}

The ENSANUT 2006 warning about the risk in which there are more than 4 million children enter between 5 to 11 years, as the combined prevalence of overweight and obesity occurs in one out of four children $[6,7]$, also revealing that on weight and obesity has continued to increase in all ages, regions and socioeconomic groups, which has led our country to take first place in the world in obesity.

In Mexico, based on information from two national sources, it was find one built in 1999 and another one in 2006, it is known that the combined prevalence of overweight and obesity has increased across the population, but particularly in the school-age population. During this period, obsesity in children increased $33 \%$ and $53 \%$ children [7].

The adverse effect and risks to health obesity early in life include both short-term physical and psychosocial problems.

Longitudinal studies that childhood obesity suggest, after 3 years of age, long-term is associated with an increased risk of obesity in adulthood and with increased morbidity, persistence of associated metabolic disorders [6].

School age and adolescence are a crucial configuration steps eating habits and other lifestyles that persist in later, with repercussions not only at this stages as to the possible impact as a risk factor, but aslo in the even in adulthood and old age.
Although there is little information regarding eating habits at school age, there are reports that report that between $7-34 \%$ of children do not eat breakfast at home before going to school [7].

Except in some private schools, public are not places where you can buy food or have spaces where they can taste. However, playtime can buy food that sells school cooperative. This name is known to the members organizations of teachers and students, staff or parents, as allowed by the regulations in force, whose role is that student's appended process of production and consumption. These cooperative are not new amd the first regulation dates from 1934, the following was issued in 1937, in 1962 appears a new, updated in 1982, which is theoretically valid. It should be mentioned that the purpose of the cooperatives are esentialy related to educational and learning teamwork, assimilation processes of production and consumption, the possibility that the products are sold at a lower price that prevails in the promotes the marked and that earnings will be used to improve the school.

Nutrition is a vital issue, as vital body processes require the supply of materials and energy to provide the necessary elements for increase and repair of body tissues [8].

One should be aware that food is one of the pillars on which health is based. For better or worse, food is the mainstay of the formation and prevention of future disease and could be atherosclerosis, hypertension, mainly diabetic and different degress of obesity that plague our society. Therefore, we must start from small, balanced meals, so our body will be healthy at the same time avoiding any childhood diseases $[11,12]$.

There are an important aspect to consider about the power system is that this can not be generalized since each individual has different nutritional needs, that is why the alimentation depend on the activities performed by an individual throughout the day, i.e., it has to be directly proportional to their activities pied otherwise fall into malnutrition. 
In nowadays society, the objectives of the child alimentation have expanded and now not only aims to achieve optimal growth and prevent malnutrition and disease occurrence caracals, but also, through the same, optimizer maturational development, establish healthy habits to prevent the onset of nutritional base diseases affecting adults, trying to get better quality of life and longevity.

As will be seen later, to secure as soon raised, we will use the Backpropagation neural network [1-4] that has some differences from traditional logic as it defines the training and classification of data, if the theory of neural networks to predict as a data set to obtain a desired output approach, particularly the prediction data.

The structure of the article is in section 2 history of the work of research, mathematical basis, neuronal structure. In stage 3 the results of the simulations of the neural system for the study of obesity with the defined variables. Section 4 is the discussion of the system compared to conventional system; Finally, section 5 shows the conclusions of the research.

\section{DATA COLLECTION (BACKGROUND)}

\section{A. Problem Statement}

In Mexico, the overweigth and obesity is a serious public health affecting school children because of all socioeconomic classes.

He believies that schools primaries National System of Educatio, which have registered more than $95 \%$ of school-age children, are a plataform that can help reverse the serious problem of overweight if it is recognized that, for now, the school is closer to being an genetic obesity environment healthy, and that part of its mission is to promote, in various educational activities, the acquisition of styles of healthy eating and physical activity. While the building has to be animed primarily at children, aslo has to involve all social actors of change factors, such as parents, family, educational institutions, community authorities and, in particular, industry producing food and beverage processing, and by advertising industry print and online [14].

\section{B. Justification}

This research grew out of the need to consider one of the possible factors that cause overweight and obesity in children, and the relationship of these with the consumptionof foods that are sold within the shool cooperatives, hygiene, food handly, prepare hygienic measures, proper sanitation requirements and frequency of food consumption.

\section{Hypothesis}

The cooperative school meets the hygenic-Nutrient, where the food consumed within the school are a determining factor in the nutritional status of children, and applying neural network techniques can predict the behavior of infant obesity $[9,10,13,15]$.

\section{Theoretical framework}

We assesed the nutrional status of students using the criteria of the Official Mexican Standard NOM-008-SSA21993, Control Nutrition, Growth and Development of Children and Adolescents and evaluation form containing: Name, Grade and Group age, weigth, height, Hips (centimeters) Waist (centimeters), BMI and food intaje [6, 7].

We evaluated the school cooperative consider through a direct observation assessment tool designed to evaluate the conditions of this, both hygenically and nutritionally about te products that are sold at recess.

Also we identified the relationship between the consumption of foods that is sold in the Cooperative School with nutritional status of students, as it is a very impactor factor for the primary students. Finally, we developed a numerical prediction system with neural network artificial Backpropagation for prediction and reinforcement of the theoretical data acquired on the job, obtained comparative test with the results of observations made, based on a sheet of data and theory a biological neuron [13, 15], and subsequently implement the system of artificial neural network with Bakcpropagation learning algorithms characterized by the equation $[1,2]$ :

$$
a^{4}=f^{4}\left(w^{4} f^{3}\left(w^{3} f^{2}\left(w^{2} f^{1}\left(w^{1} p+b^{1}\right)+b^{2}\right)+b^{3}\right)+b^{4}\right)
$$

\section{E. Neural network [1-4]}

Neural nerwork consist of a simulation of the observed properties of the biological diversity of neural systems throgh mathematical models recreated through artificial mechanisms (an integrated circuit or a computer). The aim is to ensure that the mechanies give similar answer to which are able to give to the brain.

A neural network consists of units called neurons, and each neuron receives a set of inputs through interconnections and makes an exit. This output is given by three functions:

- A propagation function, which generally consists of the sum of each input multiplied by but their interconnection. If we weight is positive, the connection is called excitatory, if negative, is called inhibitor.

- An activation function that modifies the former can not exist, being here the diffusion of the same function.

- A transfer function, which applies to the value returned by the ouput function of the neuron and generally is given by the understanding that we can give to the outputs.

\section{F. Structure}

Most scientist agree that an artificial neural network ANN [3] is very different in terms of structure of an animal brain. As brain, an ANN consist of a set of simple units massively parallel processing and connections between these units where the network intelligence.

Biologically the brain learns through the reorganization of synaptic connections between individual neurons. Similarly, RNA having a large number of virtual processors inteconnected in a simplified manner to simulate the functionality of biological neurons Figure 1. In this simulation [4], the mechanism reorganization synaptic connections biological models with weights that are adjusted during the learning phase. An ANN trained, the weights determined set of knowledge of RNA and is capable of solving a problem. 


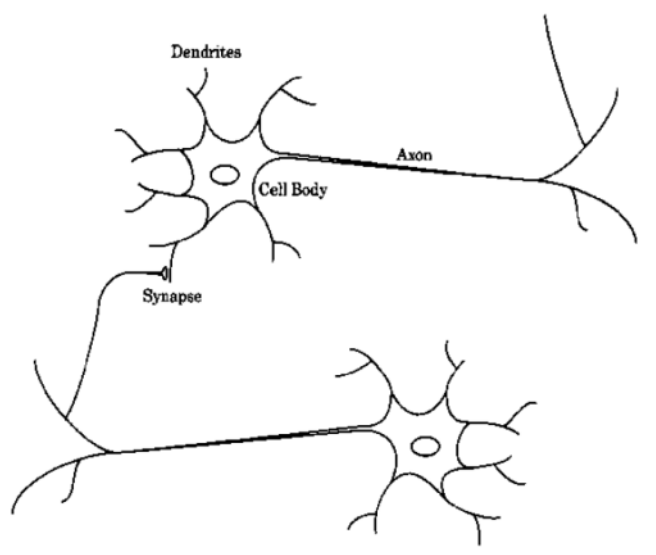

Fig. 1. A schematic drawing of biological neurons

Furthermore, each neuron is associated with a mathematical functions called transfer function. This function generatest the sugnal output from the neuron input signals. The entry of the function is the sum of all input signals but by the connection associated with that signal (Figure 2).

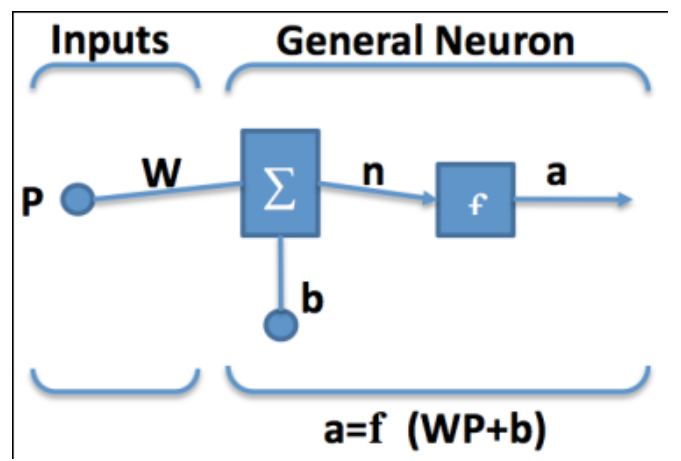

Fig. 2. Entrace of a single neuron

\section{G. The multilayer perceptron [1-4]}

It basically consists of a layer of neurons with weights and adjustable threshold; this neural system may be called a neural network because the connections exist in its entirety the Perceptron training is to determine the adjustment to be performed each neuron weight to the output error is zero.

Backpropagation algorithm is a generalization of the LMS algorithm; both algorithms perform its task of updating weights and profits based on the means square error. The network works under supervised learning and therefore requires an array of workout that will describe each output and expected output value. The structure Backpropagation neural network was shown in Figure 3.

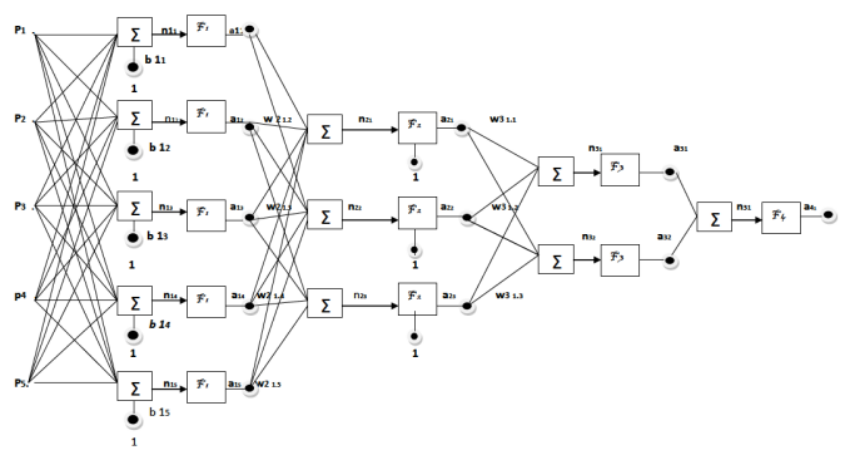

Fig. 3. Architecture Multilayer Perceptron with Backpropagation Algorithm

\section{RESULTS}

Data from the savanah of data (Table 1) should be reads as described promptly: results output with a neural network [10, 13, 15] on equal 1.9119 and 0.9326 for girl children; regarding this outcome data savannah Girls has a degree 3 of obesity and the child would have an obesity grade 2 ; dependent on the age, BMI, ICC and food intake $[11,12,13]$.

TABLE I. DATA FROM THE SAVANNAH OF DATA

\begin{tabular}{|l|l|l|l|}
\hline \multirow{2}{*}{$\begin{array}{l}\text { Ages } \\
\text { children }\end{array}$} & \multicolumn{3}{|l|}{ Prediction } \\
\cline { 2 - 4 } & Girl & Boy & Observations \\
\hline 6 & $1.8056^{\mathrm{a}}$ & 0.8640 & Obese / moderately obese \\
\hline 7 & 0.1258 & 0.8645 & $\begin{array}{l}\text { Not Obese / moderately } \\
\text { obese }\end{array}$ \\
\hline 8 & 0.8229 & 0.8647 & $\begin{array}{l}\text { Moderately obese / } \\
\text { moderately obese }\end{array}$ \\
\hline 9 & 0.5027 & 0.8641 & $\begin{array}{l}\text { Not Obese / moderately } \\
\text { obese }\end{array}$ \\
\hline 10 & 2.2126 & 0.8641 & Obese / moderately obese \\
\hline 11 & 1.7740 & 0.8641 & Obese / moderately obese \\
\hline
\end{tabular}

The following figures show numerical results of the prediction system used.

Test performed for behaviour in Women

Test $=$ [age, IMC, ICC, Food Intake $]$

Test $1=[1 ; 1 ; 3 ; 3]$; target $=\operatorname{sim}($ net, test 1$)$

Output $=1.2354$

Test $2=[12 ; 2 ; 2 ; 3] ;$ target $=\operatorname{sim}($ net, test 2$)$

Output $=0.8142$

Test $3=[12 ; 1 ; 1 ; 3] ;$ target $=\operatorname{sim}($ net, test 3$)$

Output $=0.3132$

Test $4=[2 ; 1 ; 3 ; 2] ;$ target $=\operatorname{sim}($ net, test 4$)$

Output $=0.5377$

Test $5=[8 ; 2 ; 2 ; 3] ;$ target $=$ sim $($ net, test 5$)$

Output $=0.7908$

Fig. 4. Prediction for women with different age, BMI, WHR and food intake 
Test performed for behaviour in Men

Test $=$ [age, IMC, ICC, Food Intake $]$

Test $1=[6 ; 1 ; 2 ; 3] ;$ target $=\operatorname{sim}($ net, test 1$)$

Output $=0.8665$ Not Obese

Test $2=[5 ; 1 ; 2 ; 3] ;$ target $=\operatorname{sim}($ net, test 2$)$

Output $=0.8266$ Not Obese

Test $3=[6 ; 2 ; 2 ; 2] ;$ target $=\operatorname{sim}($ net, test 3 )

Output $=0.8942$ Moderately Obese

Fig. 5. Prediction for men with different age, BMI, WHR and food intake

Test performed for behaviour in Men

Test $=$ [age, IMC, ICC, Food Intake $]$

Test $1=[1 ; 1 ; 3 ; 3] ;$ target $=\operatorname{sim}($ net, test 1$)$

Output $=0.9610$

Test $2=[12 ; 2 ; 2 ; 3] ;$ target $=\operatorname{sim}($ net, test 2$)$

Output $=0.5037$

Test $3=[2 ; 1 ; 3 ; 2] ;$ target $=\operatorname{sim}($ net, test 3$)$

Output $=0.9505$

Test $4=[8 ; 2 ; 2 ; 3] ;$ target $=\operatorname{sim}($ net, test 4$)$

Output $=0.5489$

Fig. 6. Prediction for men with different age, BMI, WHR and food intake

The following figures shows the neural behavior prediction system.

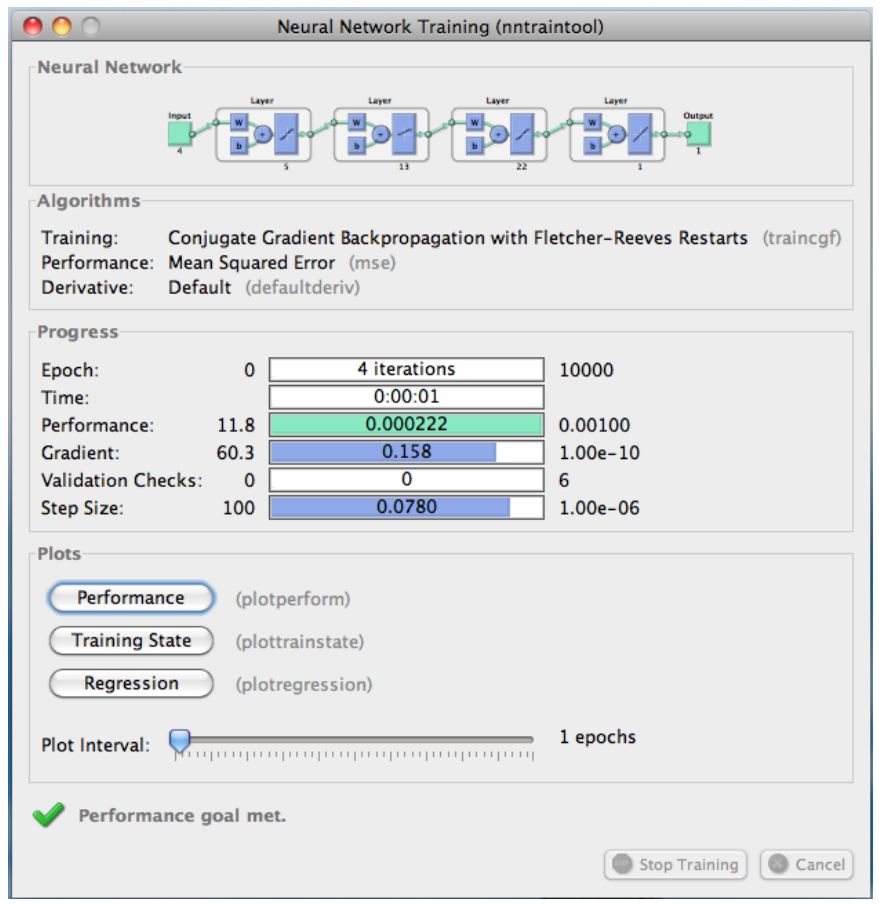

Fig. 7. Training of the ANN-BP

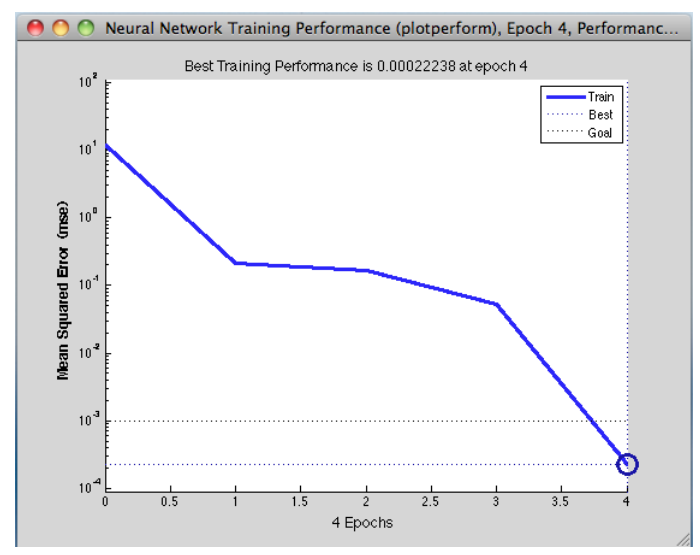

Fig. 8. Performance of the RNA-BP in sampling error

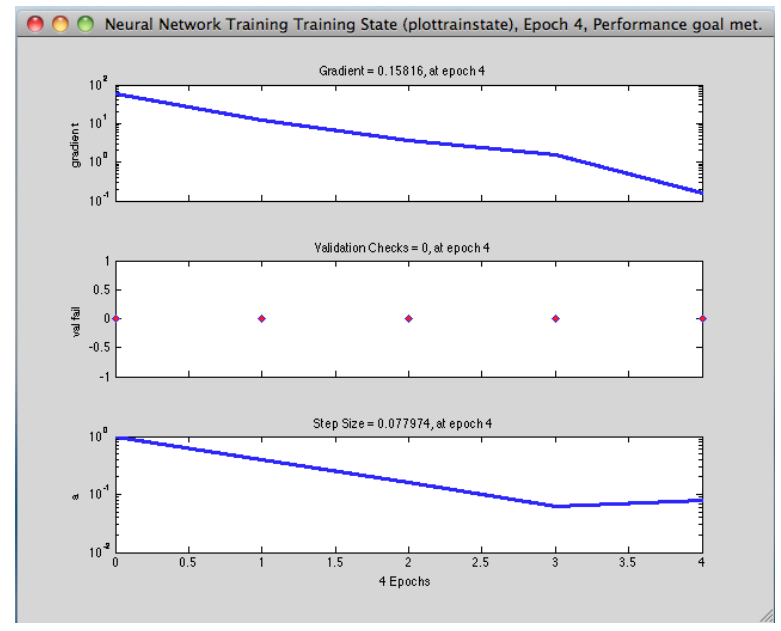

Fig. 9. State of training of the ANN-BP characterizing the system output

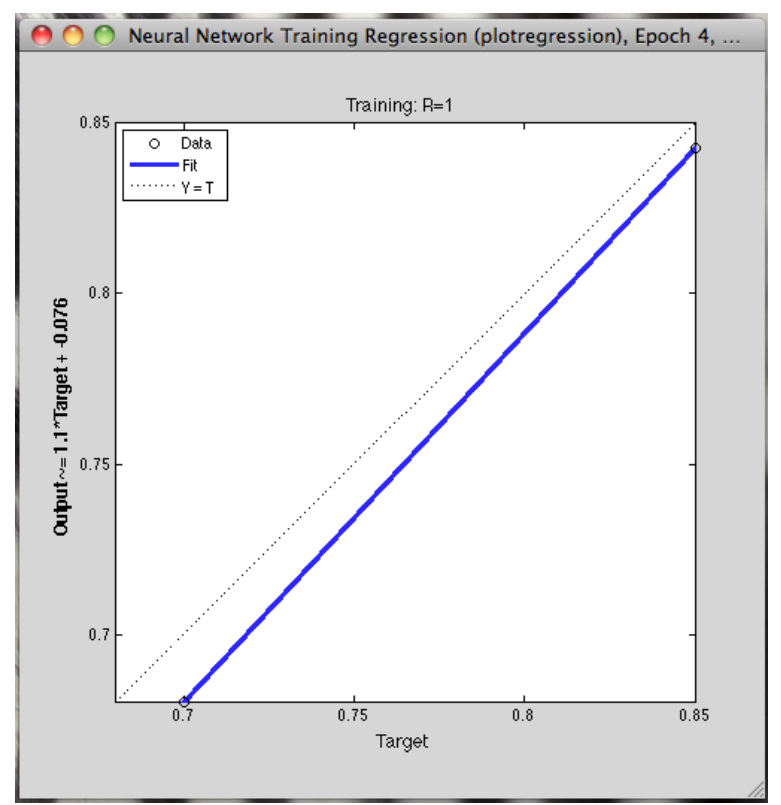

Fig. 10. Trainging Regression-BP RNA 


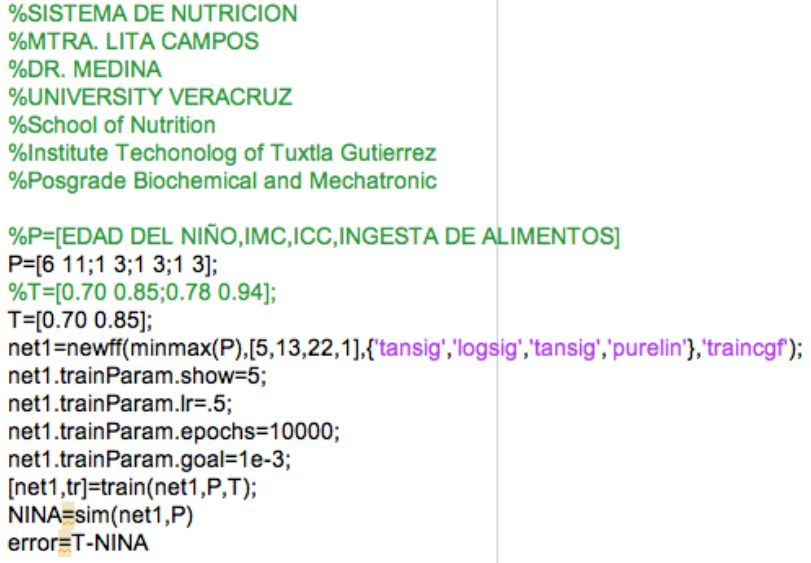

Fig. 11. Pseudocode predictive system RNA-BP development

\section{DISCUSSION}

Them results obtained with the network neuronal are of great contribution whereas a comparison of the exercise of the nutritionist without it tool and with the use of the tool increase is effectiveness, decreased the time of diagnostic of them data, reduction in the time of search in the table of nutrition to diagnose the obesity of the child school. This tool can be purchased with a system expert working group currently developing and shall submit to the test in 3 months.

\section{CONCLUSIONS}

The findings of the predictive system based on Backpropagation neural network is an effectiveness of about $99.99 \%$ of the comparative data, generating predictions on the prospects of effective school nutrition, dependent variables mentioned above characteristic. Authors and Affiliations

\section{ACKNOWLEDGMENT}

We thank the university of veracruz, faculty of nutrition for the contributions in the data sheets of children at primary level for the field tests; With a sample of 20 public schools and 10 private schools. We thank the teachers, doctors and students for their support.

\section{REFERENCES}

[1] Martin T. Hagan, Howard B. Denuth, Mark Beale, Orlando de Jesus, Neural Networks Design, $2^{\text {nd }}$ Edition, Pws Pub Co; Edición: Har/Dskt (1 de diciembre de 2014.

[2] Ryszard Tadeusiewicz and Rituparna Chaki, Exploring Neural Networks with C\#, CRC Press, 2014

[3] Scientific Books, Decision Trees, Regression and Neural Network Models with Data Minning Tools, Amazon Digital. 2015.

[4] Aurelio Uncini, Fundamentals of Adaptive Signal Processing (Signals and Communication Technology), Springer. 2015.

[5] Obesity Implications and comprehensive management. Childhood obesity. Xenical. 2013.

[6] Public health n2 V47 Mexico. Cuernavaca March / April 2005. Impact escolares a breakfast program in the prevalence of obesity and risk factors for obesity in children Sonora.

[7] Olaiz-Fernández G, Rivera-Dommarco J, Shamah-Levy T, Rojas R, Villalpando-Hernández S, Herández-Avila M, Sepúlveda-Amor J. National Health Survey and Nutricion 2006. Cuernavaca, Mexico: National Public Health Institute 2006.

[8] R Monteiro R, Azevedo I. Chronic inflammation in obesity and the metabolic syndrome. Mediators Inflamm 2010.

[9] Hung-Teng Chang, Pin-Chang Chen, Han-Chen Huang, De-Hua Lin, A Study on the Application of Neural Network to the Prediction of Weight Control, International Journal of Engineering Research and Development. Volume 5, Issue 11 (February 2013), PP. 78-85.

[10] Yu-Chuan Chang, Pei-Hsin Lin, Chun-Chang Chen, Ren-Guey Lee, Jing-Siang Huang, Tsai-Hsuan Tsai, eFurniture for home-based frailty detection using artificial neural networks and wireless sensors, Medical Engineering \& Physics, February 2013Volume 35, Issue 2, Pages 263 268.

[11] Blaum, C.S., Xue, Q.L., Michelon, E., Semba, R.D., Fried, L.P. The association between obesity and the frailty syndrome in older women: the women's health and aging studies. Journal of the American Geriatrics Society. 2005; 53:927-934.

[12] Raman Paranjape, Asha Sadanand, Multi-Agent systems for Healthcare Simulation and Modeling: Applications for System Improvement, $1^{\text {st }}$ Edition, Medical Information Science Reference. 2013.

[13] Ashley N. Gearhardt, Sonja Yokum, Eric Stice, Jennifer L. Harris and Kelly D. Brownell, Relation of obesity to neural activation in response to food commercials, Soc Cogn Affect Neurosci (2014) 9 (7): 932-938.

[14] J Liang, B E Matheson, W H Kaye and K N Boutelle, Neurocognitive correlates of obesity and obesity-related behaviors in children and adolescents, International Journal of Obesity (2014) 38, 494-506.

[15] Seyed Taghi HeydariSeyed Mohammad Taghi Ayatollahi, Najaf Zare, Comparison of Artificial Neural Networks with Logistic Regression for Detection of Obesity, Journal of Medical Systems, August 2012, Volume 36, Issue 4, pp 2449-2454. 